\title{
Localized Quantitative Characterization of Chemical Functionalization Effects on Adhesion Properties of SWNT
}

\author{
Hao Lu, Jiangnan Zhang, and Jun Lou \\ Department of Mechanical Engineering and Materials Science, Rice University, Houston, TX 77005, USA \\ Correspondence should be addressed to Jun Lou, jlou@rice.edu
}

Received 8 September 2010; Accepted 8 December 2010

Academic Editor: Teng Li

Copyright () 2011 Hao Lu et al. This is an open access article distributed under the Creative Commons Attribution License, which permits unrestricted use, distribution, and reproduction in any medium, provided the original work is properly cited.

Chemical modification of single-walled carbon nanotubes (SWNT) has been found to be an excellent method to promote SWNT dispersion, and possibly to improve interaction with matrices via covalent bonding. It is thus a quite promising technique to enhance the mechanical properties of SWNT-reinforced nanocomposites. However, the underlying mechanism of SWNT chemical functionalization effects on interfacial strength is not quantitatively understood, limiting their usefulness in the design of nanocomposites. In this work, an atomic force microscopy (AFM-) based adhesive force mapping technique combined with a statistical analysis method were developed and implemented to study adhesive interactions of small SWNT bundles functionalized by amino, epoxide, and hydroperoxide groups as compared to SDS-treated SWNT in controlled environment. Finally, the importance of such localized quantitative measurements in SWNT-reinforced nanocomposites design and fabrication was also discussed.

\section{Introduction}

Single-walled carbon nanotubes have great mechanical properties that make them excellent candidate as reinforcing agent in composites. Nanoscale fillers like SWNTs have huge potential to improve strength and fracture toughness for lightweight composite, considering their extremely small size, high aspect ratio, large interface area, and impressive theoretical strength. In particular, SWNT-reinforced polymeric nanocomposites have attracted a lot of attentions in the past decade with an increasing demand to better understand the nature of the interactions between SWNT and corresponding matrices. Ajayan and colleagues have investigated local elastic behavior of individual SWNT bundle and load transfer in epoxy composite as well as pressed pellets of composites containing SWNTs and carbonaceous soot material formed during nanotube synthesis [1]. The investigation of the carbon nanotube (CNT)/epoxy nanocomposites via transmission electron microscope (TEM) provides evidences for improved interfacial interactions between the functionalized nanotubes and the corresponding epoxy matrix [2]. Tensile tests on carbon nanotube-polystyrene composite films show that $1 \mathrm{wt} \%$ nanotube additions result in $36 \%-42 \%$ and $~ 25 \%$ increases in elastic modulus and breaking stress, respectively, indicating significant load transfer across the nanotubematrix interface [3]. Scratch resistance and scratch damage were investigated using AFM tips sliding against the SWNTreinforced nanocomposite surfaces, where nanoindentation/nanoscratch deformation and fracture behavior were carefully studied by in situ imaging of the indentation impressions/scratch tracks [4]. It was also found that polymers with a backbone structure containing aromatic rings can be used as building blocks in amphiphilic copolymers to promote increased interfacial bonding between the CNT and a polymeric matrix [5]. Li et al. have demonstrated that for SWNT-reinforced polymer composites, the covalent bonding between the nanotube and polymer matrices, crystallinity of matrices, tensile properties of the reinforcement and matrix materials, bundle effects, bundle curvature, and alignment plays important roles in mechanical reinforcement mechanisms [6]. It is now well recognized that the strength of nanotube-polymer composites depends critically on load transfers from matrix to nanotubes. Furthermore, the load transfer is closely related to the adhesion force between the matrix and the side walls of carbon nanotubes. When a composite fails, either CNTs breaks or CNTs are 
TABLE 1: List of experimental conditions.

\begin{tabular}{lcc}
\hline $\begin{array}{l}\text { Samples with different } \\
\text { functional groups }\end{array}$ & Temperature (F) & $\begin{array}{c}\text { Relative humidity } \\
(\%)\end{array}$ \\
\hline Amino & 71.9 & 5.5 \\
Epoxide & 72.5 & 7.6 \\
Hydroperoxide & 71.7 & 0 \\
SDS & 71.9 & 4.3 \\
\hline
\end{tabular}

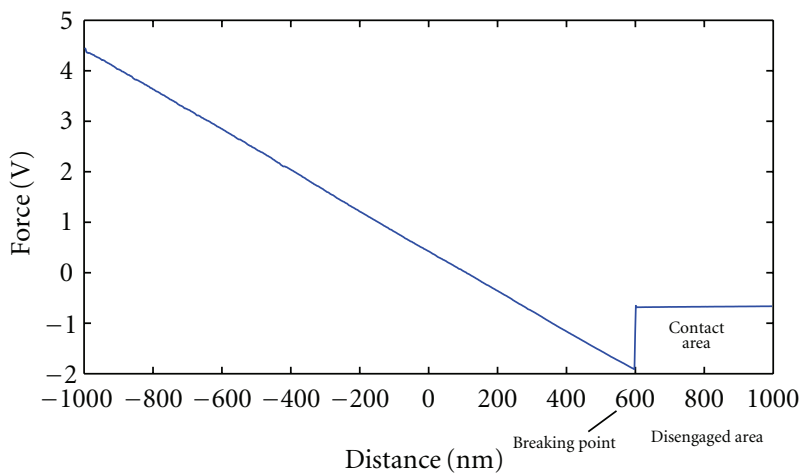

FIgURE 1: A typical force versus distance curve (the tip approaching portion of the curve was not plotted for clarity). This curve contains three parts: the disengaged area, where the AFM tip and the sample are not in contact; the contact area, where the tip and the sample are in contact; the breaking point, where the contact between the tip and the sample is just about to break.

pulled out from the matrix. The failure type depends primarily on the strength of CNTs and the interaction force between the side wall of CNTs and the matrix. The interaction force between the side walls of pristine CNTs and polymer matrix is thought to be mostly Van der Vaals interaction [7], and thus the adhesion strength between pristine CNT wall and polymer is considered to be much weaker than CNTs' intrinsic strength. The composite's strength is dominated by adhesion in this scenario, and therefore enhancement of the interaction strength at the nanotube/matrix interface is being vigorously pursued.

Various methods have been proposed to improve the adhesion strength. Introducing chemical bonds between side walls of CNTs, and the corresponding matrix by chemical modification of CNTs was a popular technique. A variety of functional groups have been applied to different CNTs and quantitatively understanding of adhesion enhancement by these functional groups is of great importance. Poggi et al. $[8,9]$ have studied the adhesion force between AFM probes and CNTs paper with different function groups. While their results shed lights into the interfacial interactions between CNTs and AFM probes, the scenario might be quite different in real composite where CNTs are typically dispersed and distributed. Therefore, we seek to measure the adhesion properties of a small bundle of SWNTs which is more realistic for composite application compared to densely packed CNTs in CNT paper [8, 9]. SWNTs with functionalization groups of amino $\left(-\mathrm{NH}_{2}\right)$, epoxide (-COC), hydroperoxide (-OOH),

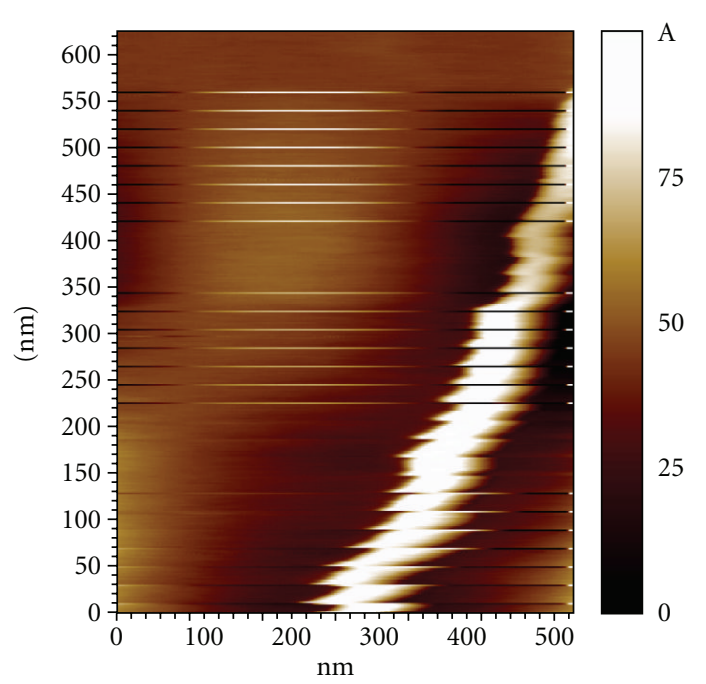

(a)

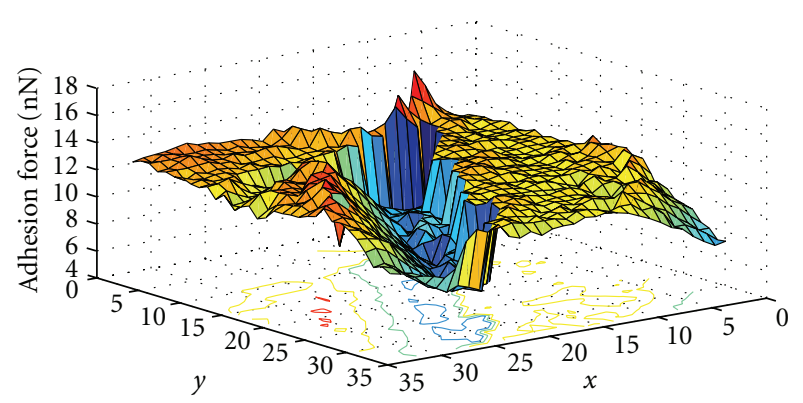

(b)

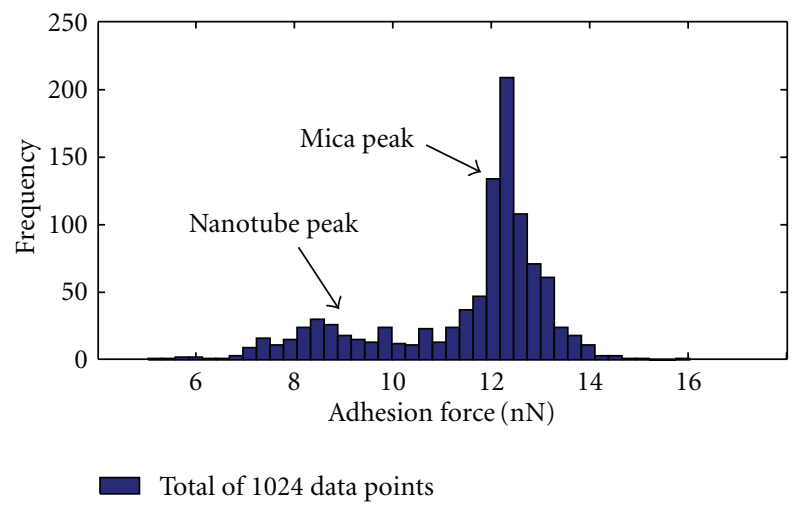

(c)

FIGURE 2: (a) AFM topography and spectroscopy image; (b) Adhesion force mapping on amino-functionalized SWNTs; (c) Histogram with two peaks shows the adhesion forces on aminofunctionalized SWNTs and the mica substrate, respectively.

and SWNTs physically wrapped with Sodium Dodecyl Sulfate (SDS) were studied in this work.

\section{Materials and Experimental Procedures}

Four types of SWNT samples with different functional groups and treatments $\left(-\mathrm{NH}_{2},-\mathrm{COC},-\mathrm{OOH}\right.$, and SDS) were obtained from NanoRidge Inc. (Houston,TX) and 
TABLE 2: Adhesion forces normalized on mica. CNT with amino function group and SDS-wrapped CNT have larger adhesion force than the other two.

\begin{tabular}{lccc}
\hline Functional groups & $\begin{array}{c}\text { Adhesion on nanotube } \\
(\text { Unit: } \mathrm{nN})\end{array}$ & $\begin{array}{c}\text { Adhesion on mica } \\
(\text { Unit: } \mathrm{nN})\end{array}$ & $\begin{array}{c}\text { Normalized adhesion on } \\
\text { nanotubes }\end{array}$ \\
\hline Amino & $8.71 \pm 1.05$ & $12.35 \pm 0.65$ & 0.70 \\
Epoxide & $4.20 \pm 1.25$ & $8.22 \pm 0.38$ & 0.51 \\
Hydroperoxide & $7.77 \pm 1.28$ & $15.61 \pm 0.88$ & 0.50 \\
SDS & $5.00 \pm 0.70$ & $6.98 \pm 0.57$ & 0.72 \\
\hline
\end{tabular}

were tested in a our experiments. SWNTs were dispersed in Dimethylformamide (DMF) and sonicated for 5 minutes. One drop of upper layer of the sonicated solution was deposited on a freshly cleaved mica surface and dried in ambient condition. A PicoPlus 5500 AFM (Agilent Technologies) was used to obtain topography image and the corresponding adhesion force mapping. In order to obtain more accurate adhesion force mapping, the square scan area was divided into 32 by 32 subareas. A force versus distance (FD) curve was then collected in each subarea, and the corresponding adhesion force was acquired from the FD curve. The AFM cantilever used was calibrated using a standard reference cantilever method [10], and the measured spring constant is $0.043 \mathrm{~N} / \mathrm{m}$, which is very close to the manufacturer's specifications. The same AFM cantilever was used in all experiments to exclude variations caused by using different tips. Humidity of the testing environment was controlled using an environmental isolation chamber. The topography images were first obtained for every sample, and a single or a small bundle of nanotubes was then identified based on the topography information. After a series of zooming in onto the identified nanotubes (by reducing the AFM scan size), adhesion force mapping was obtained by collecting 1,024 force versus distance curves according to a 32-by-32 matrix configuration in the final scanned area. A typical FD curve is shown in Figure 1. From the FD curve, the adhesion force is defined by the difference between the force at disengaged region and the force at the breaking point. The temperature and relative humidity were carefully controlled and monitored during the experiment as listed in Table 1. The temperature variation was quite small during our experiments, and the relative humidity was maintained to be below $10 \%$ using dry nitrogen.

\section{Results and Discussion}

After locating a single SWNT or a small SWNT bundle, the above-mentioned FD spectroscopy mapping was collected on each sample. The typical mapping size was generally below $1 \mu \mathrm{m}$ by $1 \mu \mathrm{m}$. A typical adhesion force mapping and the associated topography image for amino-functionalized SWNTs are shown in Figure 2. It should be noted that the features in the adhesion force mapping image (Figure 2(b)) nearly replicates the AFM topography image (Figure 2(a)), with the portion of lower adhesion forces in Figure 2(b) correspond to higher topography features in Figure 2(a) showing the location of the amino-functionalized SWNT bundle. Interestingly, there still exists a slight pattern mismatch which might be caused by the hysteresis of the AFM scanner and also friction-induced small deviation between the point where spectroscopy was collected and the point where topography was measured [11]. The discontinuities of CNT topography image in Figure 2(a) seem to support this hypothesis. The associated histogram for the measured adhesive forces was also plotted in Figure 2(c). Two adhesion force peaks with one on nanotube and another on mica were very evident although the mica peak dominated over nanotube peak as shown in Figure 2(c). However, for most of other adhesion experiments, the nanotube peaks cannot be clearly identified from the histogram. For the purpose of separating adhesion forces on nanotubes from that on the mica substrate, an optimal global threshold was found using the Otsu's method [12] for each experiment. Adhesion forces below the threshold were thought to be the adhesion between the tip and the SWNTs while forces larger than the threshold were identified as the adhesion between the tip and the mica. The adhesion force mapping images of amino-functionalized SWNTs before and after the application of the Otsu's method were shown in Figure 3. Adhesion forces below the carefully chosen threshold were considered to be adhesion on SWNTs as shown in white in Figure 3(b), while forces larger than the threshold were thought to be the adhesion on mica substrate as shown in black in the same figure. Average adhesion forces and standard deviations could then be calculated and are summarized in Table 2. Although it is reasonable to assume that the adhesion forces between the AFM tip and the mica substrates should be identical since the same AFM tip was used to probe relatively homogeneous mica substrates, we have observed dissimilar absolute adhesion forces in different experiments. This has been attributed to the variations in testing environments. In order to be able to make more meaningful comparisons of the results obtained from different experiments, the adhesion forces measured on nanotubes were also normalized against the adhesion forces on mica. As shown in Table 2, the measured adhesion forces between the AFM tip and the nanotubes are typically less than $10 \mathrm{nN}$. Compared with the individual bond strength measured by Friddle et al. [13], the adhesion force measured in our experiments came from breaking of tens of bonds at the same time.

The height of the amino-functionalized nanotubes was also estimated from the topography image (Figure 4(a)). Figure 4(b) is the cross sectional line of the SWNTs shown in Figure 4(a). The height of it is determined to be $6.8 \mathrm{~nm}$, 


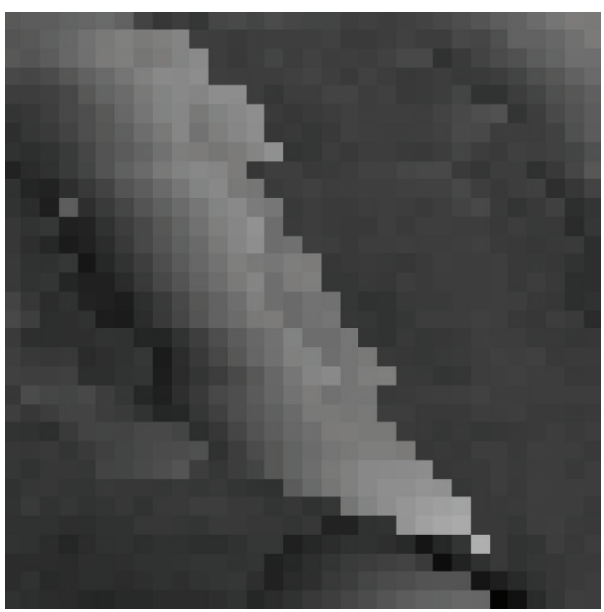

(a)

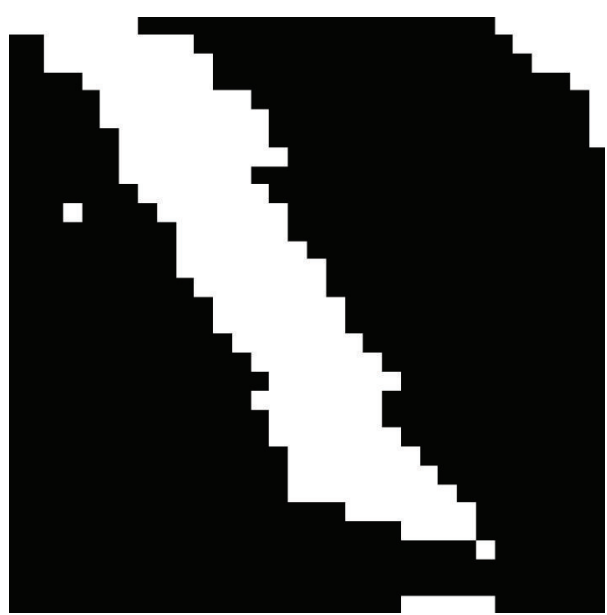

(b)

FIGURE 3: (a) Grey image of adhesion mapping for amino-functionalized CNT. (b) Black and white image after finding a global threshold with Otsu's method. White area represents CNT, and black part represents mica.

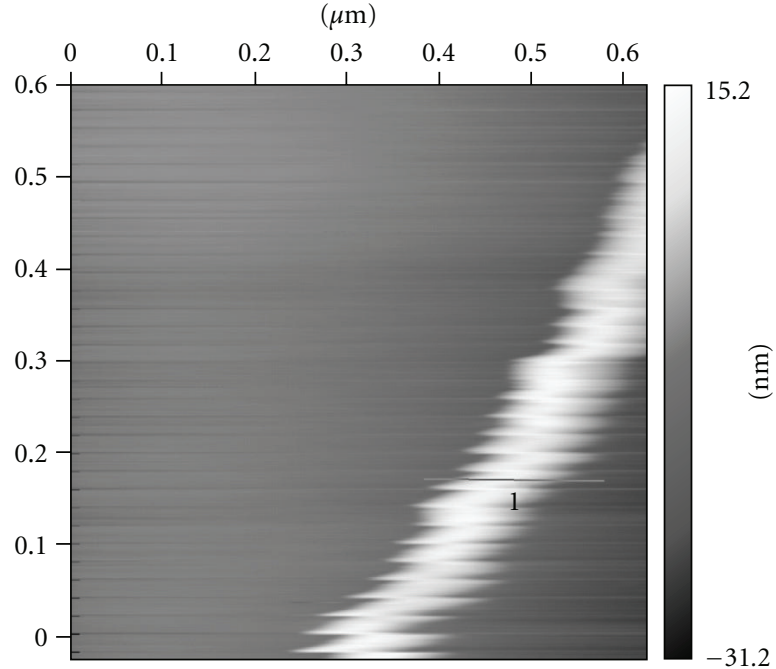

(a)

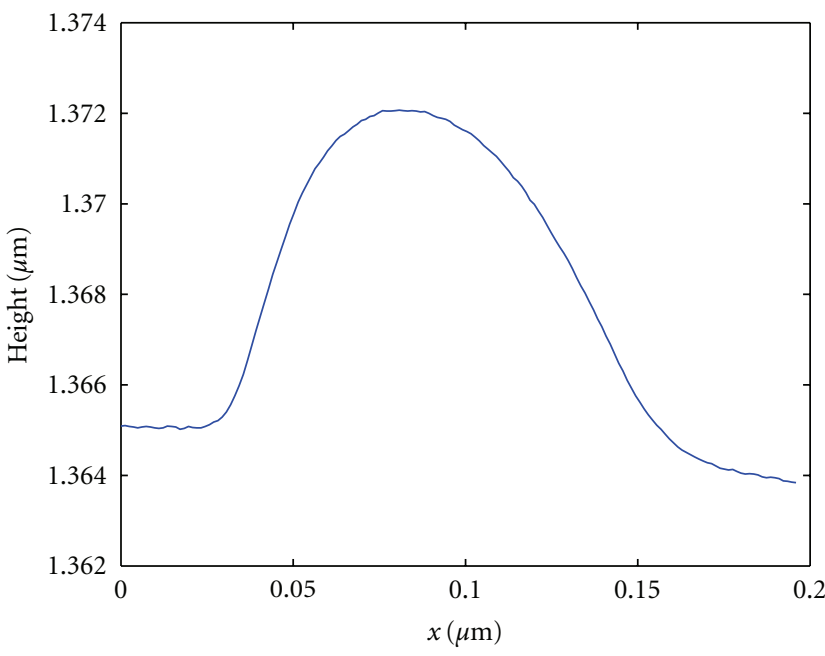

(b)

FIgURE 4: Topography image of amino-functionalized nanotubes. (b) Cross section of the SWNTs. $x$-axis is the lateral dimension while $y$-axis is the height in nanometer.

indicating that this is a small SWNT bundle with 4-6 single nanotubes. Other SWNT samples typically have height of $\sim 10-14 \mathrm{~nm}$ with more than ten single nanotubes.

When comparing the normalized adhesion forces for different functionalized nanotubes (Table 2), it is found that the adhesion forces of amino-functionalized nanotube and SDSwrapped nanotubes are larger than those of epoxide group terminated and hydroperoxide-terminated nanotubes. Such localized quantitative measurements of adhesion properties of small SWNT bundles provide an effective tool to assess the effectiveness of different chemical treatments in improving the adhesions between CNTs and matrix due to its realistic sample morphology and fast turn-around time as compared to costly full production and evaluation cycle of producing nanocomposites. The AFM tips can be further treated with different matrix material coating or by attaching the appropriate moieties similar to the backbone structures of corresponding polymeric matrices. These improvements will be pursued in the future studies and are thus beyond the scope of current work.

\section{Conclusion}

A localized quantitative method to measure adhesion properties of single-walled nanotubes treated with different functional groups has been reported in this paper. By carefully 
analyzing collected adhesive force mapping data using the Otsu's method, we have successfully identified the adhesion forces between the AFM tip and SWNTs. The normalized adhesion on SWNTs with different functional groups is considered to be a very useful parameter to evaluate the effects of functionalization on interfacial interactions between matrices and SWNTs in nanocomposite applications.

\section{Authors' Contribution}

H. Lu and J. Zhang contributed equally to this work.

\section{Acknowledgments}

This work was supported by a National Science Foundation Grant CMMI no. 0800896, an Air Force Research Laboratory Grant AFRL no. FA8650-07-2-5061, and a Welch Foundation Grant no. C-1716. The authors would like to thank Dr. Jiang Zhu (NanoRidge, Inc.) and Dr. Barrera (Rice University) for providing the SWNT materials studied in this work.

\section{References}

[1] P. M. Ajayan, L. S. Schadler, C. Giannaris, and A. Rubio, "Single-walled carbon nanotube-polymer composites: strength and weakness," Advanced Materials, vol. 12, no. 10, pp. 750-753, 2000.

[2] F. H. Gojny, J. Nastalczyk, Z. Roslaniec, and K. Schulte, "Surface modified multi-walled carbon nanotubes in CNT/epoxycomposites," Chemical Physics Letters, vol. 370, no. 5-6, pp. 820-824, 2003.

[3] D. Qian, E. C. Dickey, R. Andrews, and T. Rantell, "Load transfer and deformation mechanisms in carbon nanotubepolystyrene composites," Applied Physics Letters, vol. 76, no. 20, pp. 2868-2870, 2000.

[4] X. Li, H. Gao, W. A. Scrivens et al., "Nanomechanical characterization of single-walled carbon nanotube reinforced epoxy composites," Nanotechnology, vol. 15, no. 11, pp. 1416$1423,2004$.

[5] M. Yang, V. Koutsos, and M. Zaiser, "Interactions between polymers and carbon nanotubes: a molecular dynamics study," Journal of Physical Chemistry B, vol. 109, no. 20, pp. 1000910014, 2005.

[6] X. Li, H. Gao, W. A. Scrivens et al., "Reinforcing mechanisms of single-walled carbon nanotube-reinforced polymer composites," Journal of Nanoscience and Nanotechnology, vol. 7, no. 7, pp. 2309-2317, 2007.

[7] K. Liao and S. Li, "Interfacial characteristics of a carbon nanotube-polystyrene composite system," Applied Physics Letters, vol. 79, no. 25, pp. 4225-4227, 2001.

[8] M. A. Poggi, L. A. Bottomley, and P. T. Lillehei, "Measuring the adhesion forces between alkanethiol-modified AFM cantilevers and single walled carbon nanotubes," Nano Letters, vol. 4, no. 1, pp. 61-64, 2004.

[9] M. A. Poggi, P. T. Lillehei, and L. A. Bottomley, "Chemical force microscopy on single-walled carbon nanotube paper," Chemistry of Materials, vol. 17, no. 17, pp. 4289-4295, 2005.

[10] M. Tortonese and M. Kirk, "Characterization of applicationspecific probes for SPMs," in Micromachining and Imaging, Proceedings of SPIE, pp. 53-60, San Jose, Calif, USA, February 1997.
[11] J. H. Hoh and A. Engel, "Friction effects on force measurements with an atomic force microscope," Langmuir, vol. 9, no. 11, pp. 3310-3312, 1993.

[12] N. Otsu, "A threshold selection Method from gray-level histograms," IEEE Transactions on Systems, Man, and Cybernetics, vol. 9, no. 1, pp. 62-66, 1979.

[13] R. W. Friddle, M. C. Lemieux, G. Cicero et al., "Single functional group interactions with individual carbon nanotubes," Nature Nanotechnology, vol. 2, no. 11, pp. 692-697, 2007. 

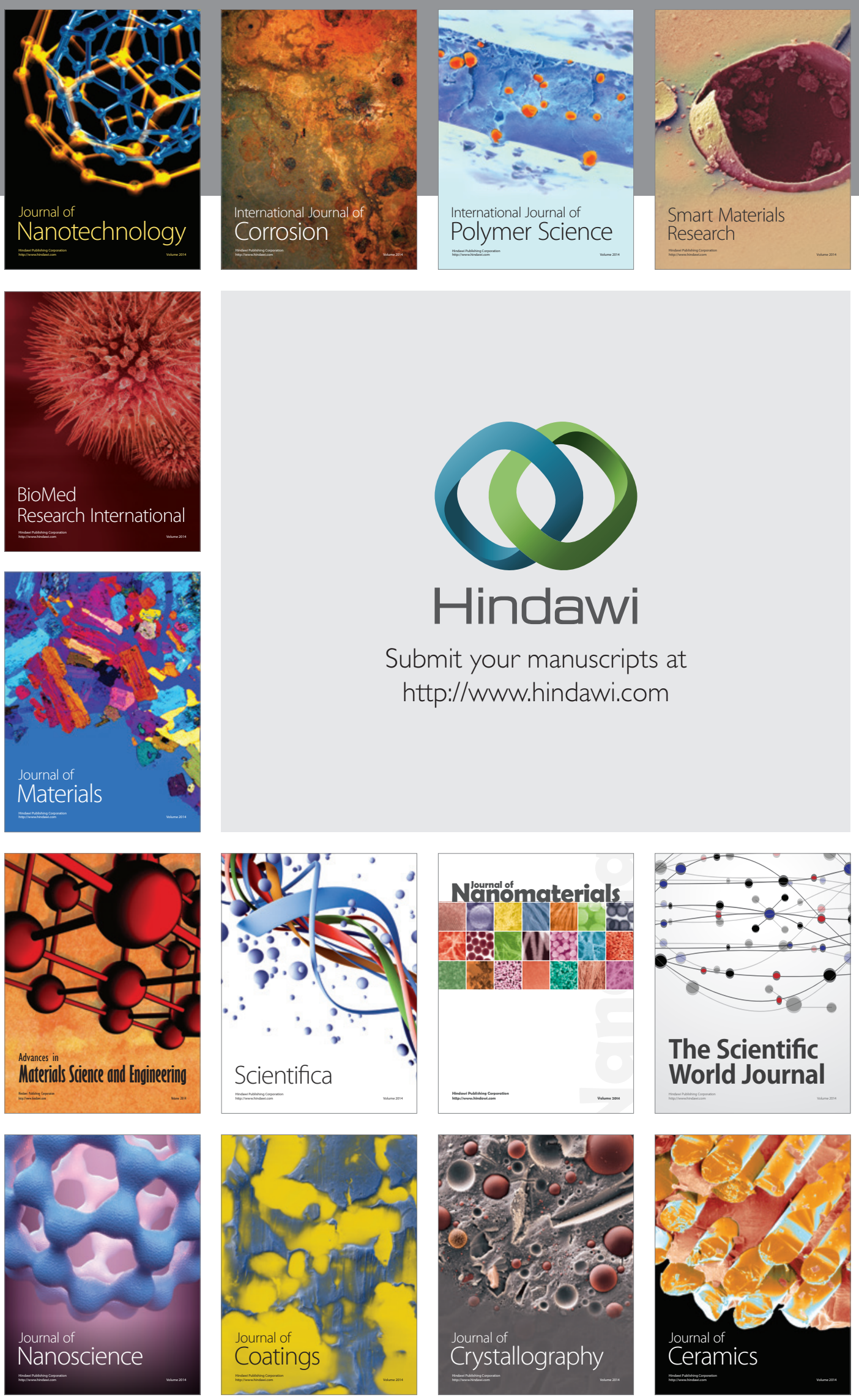

The Scientific World Journal

Submit your manuscripts at

http://www.hindawi.com

\section{World Journal}

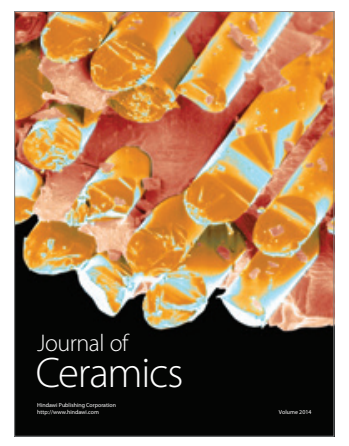

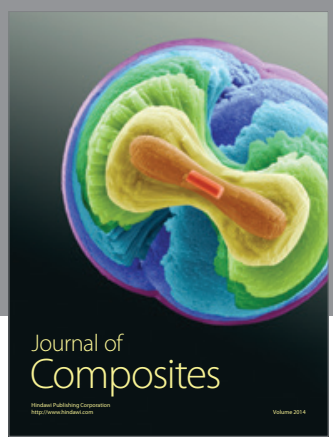
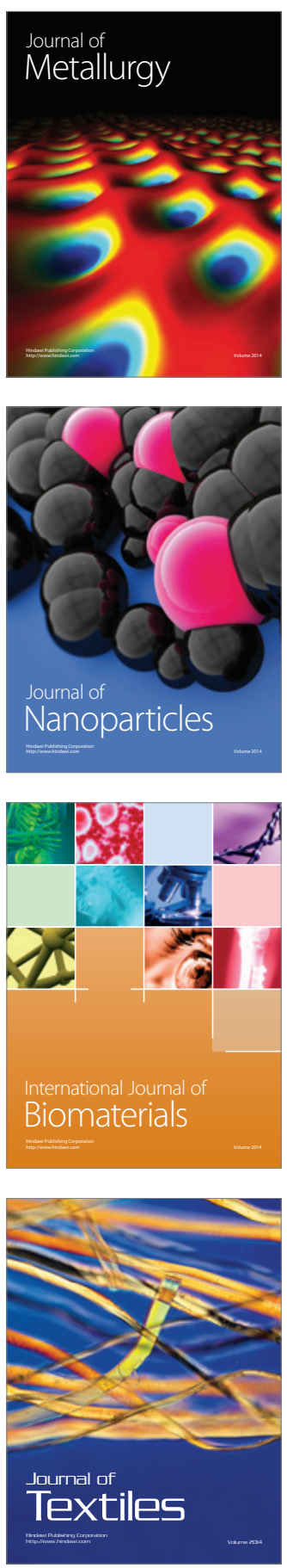